\title{
AN ERROR ESTIMATE \\ OF THE LEAST SQUARES FINITE ELEMENT METHOD FOR THE STOKES PROBLEM IN THREE DIMENSIONS
}

\author{
CHING LUNG CHANG
}

\begin{abstract}
In this paper we are concerned with the Stokes problem in three dimensions (see recent works of the author and B. N. Jiang for the two-dimensional case). It is a linear system of four PDEs with velocity $\underline{u}$ and pressure $p$ as unknowns. With the additional variable $\underline{\omega}=\operatorname{curl} \underline{u}$, the second-order problem is reduced to a first-order system. Considering the compatibility condition $\operatorname{div} \underline{\omega}=0$, we have a system with eight first-order equations and seven unknowns. A least squares method is applied to this extended system, and also to the corresponding boundary conditions. The analysis based on works of $\mathrm{Ag}$ mon, Douglis, and Nirenberg; Wendland; Zienkiewicz, Owen, and Niles; etc. shows that this method is stable in the $h$-version. For instance, if we choose continuous piecewise polynomials to approximate $\underline{u}, \underline{\omega}$, and $p$, this method achieves optimal rates of convergence in the $H^{1}$-norms.
\end{abstract}

\section{INTRODUCTION}

Let $\Omega$ be an open bounded and connected subset of $\mathbb{R}^{3}$ with a smooth boundary $\Gamma$. Let $f \in\left[L^{2}(\Omega)\right]^{3}$ be a given function representing the body force. The Stokes problem can be posed as

$$
\begin{cases}-\nu \Delta \underline{u}+\operatorname{grad} p=\underline{f} & \text { in } \Omega, \\ \operatorname{div} \underline{u}=0 & \text { in } \Omega, \\ \underline{u}=0 & \text { on } \Gamma,\end{cases}
$$

where $\underline{u}, p$ with $(p, 1)=0$, and $\nu$ are respectively velocity, pressure, and kinematic viscosity (constant), all of which are assumed to be nondimensionalized.

Over the past two decades many engineers and mathematicians have studied the above problem. The mixed Galerkin method solves this problem successfully. In most cases the elements are required to satisfy a saddle point condition $[4,5,8,9,22]$, which is not necessary for our method.

Received by the editor March 16, 1992 and, in revised form, October 12, 1992.

1991 Mathematics Subject Classification. Primary 65N30, 35F15.

This work was performed when the author was an OAI/CWRU Summer Faculty fellow participant at NASA Lewis Research Center, Cleveland, OH (1991), and was revised at the University of Texas at Arlington (1992) and at Wright-Patterson Air Force Base, Dayton, OH (1993). 
Introducing $\underline{\omega}=\operatorname{curl} \underline{u}$, we can transform (1.1) into the first-order system

$$
L \underline{U}=\left[\begin{array}{c}
\nu \operatorname{cur} \underline{\omega}+\operatorname{grad} p \\
-\operatorname{div} \underline{\omega} \\
\nu \operatorname{curl} \underline{u}-\nu \underline{\omega} \\
-\operatorname{div} \underline{u}
\end{array}\right]=\left[\begin{array}{c}
\frac{f}{0} \\
0 \\
0 \\
0 \\
0
\end{array}\right] \quad \text { in } \Omega
$$

and the boundary condition

$$
R \underline{U}=\left[\begin{array}{ccccccc}
1 & 0 & 0 & 0 & 0 & 0 & 0 \\
0 & 1 & 0 & 0 & 0 & 0 & 0 \\
0 & 0 & 1 & 0 & 0 & 0 & 0 \\
0 & 0 & 0 & n_{1} & n_{2} & n_{3} & 0
\end{array}\right] \underline{U}=\underline{0} \quad \text { on } \Gamma,
$$

where $\underline{U}=[\underline{u}, \underline{\omega}, p]^{t}$. The above system has been weighted, which is required by the analysis in the following sections. The relation $\operatorname{div} \underline{\omega}=0$ is the compatibility condition; without it, the numerical scheme may not be convergent.

The boundary condition $\underline{u}=\underline{0}$ on $\Gamma$ implies that the tangential derivatives of $u_{i}$ vanish, or $\nabla u_{i} \times \underline{n}=\underline{0}$ for $i=1,2,3$ and

$$
\begin{aligned}
\underline{\omega} \cdot \underline{u} & =\left(\frac{\partial u_{3}}{\partial y}-\frac{\partial u_{2}}{\partial z}\right) n_{1}+\left(\frac{\partial u_{1}}{\partial z}-\frac{\partial u_{3}}{\partial x}\right) n_{2}+\left(\frac{\partial u_{2}}{\partial x}-\frac{\partial u_{1}}{\partial y}\right) n_{3} \\
& =\left(\frac{\partial u_{3}}{\partial y} n_{1}-\frac{\partial u_{3}}{\partial x} n_{2}\right)+\left(\frac{\partial u_{2}}{\partial x} n_{3}-\frac{\partial u_{2}}{\partial z} n_{1}\right)+\left(\frac{\partial u_{1}}{\partial z} n_{2}-\frac{\partial u_{1}}{\partial y} n_{3}\right) \\
& =0 .
\end{aligned}
$$

The least squares method relaxes the boundary conditions and the exact divergence-free condition, so that the elements require less restriction. For example, if all of the $\underline{u}, \underline{\omega}$, and $p$ are allowed to be approximated by piecewise linear functions in $H^{1}(\Omega)$, we will show that the method achieves an optimal rate of convergence.

Weighted least squares methods were used by Bramble, Nitsche, Schatz, Fix, Gunzburger, Nicolaides, Oden, Carey, Zienkiewicz, and many others [7, 15] in $[2,12]$. In this paper we are going to apply the theory of Agmon-DouglisNirenberg-type first-order linear systems to the weighted least squares methods. The work of Aziz, Kellogg, Stephens, and Wendland [2, 23] gave a general theory for this method. Jiang, Povinelli, and Chang [18-19] have successfully transformed the Stokes problem into a first-order system in a two-dimensional region and then treated it by a least squares method.

In this paper we will present not only the numerical least squares scheme, but also derive error estimates in the three-dimensional case.

\section{NOTATION AND FORMULATION OF THE PROBLEM}

Throughout this paper, we will employ standard notation for Sobolev spaces and their associated norms $[14,22]$. We let $H^{m}(\Omega)$ denote the Sobolev space of functions having square integrable derivatives of order up to $m$ over $\Omega$,

$$
H^{m}(\Omega)=\left\{v \in L^{2}(\Omega) ; \partial^{\alpha} v \in L^{2}(\Omega) \text { for }|\alpha| \leq m\right\},
$$


where $\alpha=\left(\alpha_{1} \alpha_{2} \alpha_{3}\right), \partial^{\alpha} v=\partial^{|\alpha|} v / \partial x^{\alpha_{1}} \partial y^{\alpha_{2}} \partial z^{\alpha_{3}},|\alpha|=\alpha_{1}+\alpha_{2}+\alpha_{3}$. We define the inner product and the norm in $H^{m}(\Omega)$ as

$$
(u, v)_{m}=\sum_{|\alpha| \leq m} \int_{\Omega} \partial^{\alpha} u \cdot \partial^{\alpha} v
$$

and

$$
\|u\|_{m}^{2}=(u, u)_{m} .
$$

The space $H_{0}^{m}(\Omega)$ is the closure of $\mathscr{D}(\Omega)$ for the norm $\|\cdot\|_{m}$, where $\mathscr{D}(\Omega)$ is the linear space of functions infinitely differentiable and with compact support on $\Omega$. We denote by $H^{-m}(\Omega)$ the dual space of $H_{0}^{m}(\Omega)$ normed by

$$
\|u\|_{-m}=\sup \frac{|(u, v)|}{\|v\|} \text { over } v \in H_{0}^{m}(\Omega) \text { and } v \not \equiv 0 .
$$

The trace operator $\gamma_{0}: H^{1}(\Omega) \rightarrow L^{2}(\Gamma)$ is a bounded linear operator agreeing with the restriction operator $u \mapsto u / \Gamma$ for continuous functions on $\bar{\Omega}$. The kernel of $\gamma_{0}$ is $H_{0}^{1}(\Omega)$, and the image is denoted by $H^{1 / 2}(\Gamma)$, which is also a Hilbert space; we define its norm by

$$
\|u\|_{1 / 2, \Gamma}=\left\{\inf \|v\|_{1} ; v \in H^{1}(\Omega) \text { and } \gamma_{0} U=u \text { on } \Gamma\right\} .
$$

The trace inequality shows that there exists $c>0$ independent of $v$ such that

$$
\left\|\gamma_{0} v\right\|_{1 / 2, \Gamma} \leq c\|v\|_{1} \quad \text { for any } v \in H^{1}(\Omega) \text {. }
$$

We define the function space

$$
V=\left\{\underline{v} \in\left[H^{1}(\Omega)\right]^{7}\right\} .
$$

Following the work of Bramble and Scott [6], we will use a finite-dimensional subspace $V_{r}^{h} \in V$ of functions to approximate our solutions. The parameter $h$, which represents a mesh spacing, is used to indicate the approximation property of $V_{r}^{h}$. We say that $V_{r}^{h}$ approximates optimally with respect to $r$ if for every $\underline{v} \in V \cap\left[H^{r+1}(\Omega)\right]^{7}$ there exists $\underline{v}^{h} \in V_{r}^{h}$ such that

$$
h\left\|\underline{v}-\underline{v}^{h}\right\|_{1}+\left\|\underline{v}-\underline{v}^{h}\right\|_{0} \leq C h^{r+1}\|\underline{v}\|_{r+1},
$$

where the positive constant $C$ is independent of $\underline{v}$ and $h$.

We then define the least squares quadratic functional

$$
J(\underline{v})=\int_{\Omega}\left[L \underline{v}-\left(\frac{f}{0}\right)\right] \cdot\left[L \underline{v}-\left(\frac{f}{\underline{0}}\right)\right]+h^{-1} \int_{\Gamma} R \underline{v} \cdot R \underline{v} \quad \text { for } \underline{v} \in V .
$$

If $\underline{U}$ minimizes $J(\underline{v})$ over $\underline{v} \in V$, it is easy to see that

$$
\int_{\Omega} L \underline{U} \cdot L \underline{v}+h^{-1} \int_{\Gamma} R \underline{U} \cdot R \underline{v}=\int_{\Omega}\left(\frac{f}{\underline{0}}\right) \cdot L \underline{v} \quad \text { for any } \underline{v} \in V,
$$

so a solution of (1.2) is also a solution of (2.9), and a sufficiently smooth solution of (2.9) is also a solution of (1.2).

A finite element approximation to the solution of (1.2) or (2.9) is defined as a solution of the problem

$$
\operatorname{Min} J\left(\underline{v}^{h}\right) \quad \text { over } \underline{v}^{h} \in V_{r}^{h} .
$$


Similarly to (2.9), the solution $\underline{U}^{h}$ of (2.10) satisfies the corresponding finite algebraic equations

$$
\int_{\Omega} L \underline{U}^{h} \cdot L \underline{v}^{h}+h^{-1} \int_{\Gamma} R \underline{U}^{h} \cdot R \underline{v}^{h}=\int_{\Omega}\left(\frac{f}{\underline{0}}\right) \cdot L \underline{v}^{h} \quad \text { for any } \underline{v}^{h} \in V_{r}^{h} .
$$

Once a basis for $V_{r}^{h}$ is chosen, (2.11) becomes a symmetric linear algebraic system. Moreover, in $\S 4$ we will show that this algebraic system is also positive definite.

\section{THE A PRIORI ESTIMATES}

In this section we consider an auxiliary elliptic system obtained from the system (1.2) by adding another unknown. The a priori inequality associated with this "enriched" system is important in the derivation of our error estimate. The "enriched" set of unknowns is $\underline{\mathscr{U}}=[\underline{u}, k, \underline{\omega}, p]^{t}$, where $k$ is a new variable that plays a role similar to that of a slack variable in linear programming. The enriched differential system is defined in terms of the operator $L_{e}$ by

$$
L_{e} \underline{\mathscr{U}}=\left[\begin{array}{c}
\nu \operatorname{curl} \underline{\omega}+\operatorname{grad} p \\
-\operatorname{div} \underline{\omega} \\
\nu \operatorname{curl} \underline{u}+\operatorname{grad} k-\nu \underline{\omega} \\
-\operatorname{div} \underline{u}
\end{array}\right]=\underline{F} \quad \text { in } \Omega,
$$

while the enriched boundary conditions are given in terms of the boundary operator $R_{e}$ by

$$
\begin{aligned}
R_{e} \underline{\mathscr{U}} & =\left[\begin{array}{cccccccc}
1 & 0 & 0 & 0 & 0 & 0 & 0 & 0 \\
0 & 1 & 0 & 0 & 0 & 0 & 0 & 0 \\
0 & 0 & 1 & 0 & 0 & 0 & 0 & 0 \\
0 & 0 & 0 & 0 & n_{1} & n_{2} & n_{3} & 0
\end{array}\right] \underline{\mathscr{U}} \\
& =\left[\begin{array}{ccc}
\multicolumn{7}{c}{u_{1}} \\
u_{2} \\
u_{3} \\
n_{1} \omega_{1}+ & n_{2} \omega_{2} & +n_{3} \omega_{3}
\end{array}\right]=\underline{0} \text { on } \Gamma .
\end{aligned}
$$

If $\int_{\Omega} p=\int_{\Omega} k=0$ and the compatibility conditions on the data, $\int_{\Omega} f_{4}=$ $\int_{\Omega} f_{8}=0$, are satisfied, the boundary value problem defined by $(3.1),(3.2)$ is well posed, and the a priori inequality associated with this problem gives rise to our desired inequality for the Stokes problem. Taking the div of both sides of equations 5 through 7 in (3.1) yields $\Delta k=0$; evaluating the normal component of the vector consisting of the same three rows of $L_{e} \underline{\mathscr{U}}$ anywhere on the boundary yields $\frac{\partial k}{\partial n}=0,{ }^{1}$ and together with $\int_{\Omega} k=0$ this finally gives $k \equiv 0$. Equation (3.1) can thus be rewritten as

$$
L_{e} \underline{\mathscr{U}}=A \underline{\mathscr{U}}_{x}+B \underline{\mathscr{U}}_{y}+C \underline{\mathscr{U}}_{z}+D \underline{\mathscr{U}}=\underline{F},
$$

where $A, B, C$, and $D$ are $8 \times 8$ constant matrices:

$$
A=\left[\begin{array}{ll}
A_{1} & A_{2} \\
A_{3} & A_{4}
\end{array}\right]
$$

\footnotetext{
${ }^{1} \nabla k \cdot \underline{n}=\nu(\underline{\omega}-\operatorname{curl} \underline{u}) \cdot \underline{n}=\nu\left(\underline{\omega} \cdot \underline{n}-\sum_{i=1}^{3}\left[\nabla u_{i} \times \underline{n}\right]_{i}\right)=\nu(0-0)=0$, since $\underline{\omega} \cdot \underline{n}=0$ and $\underline{u}=\underline{0}$ on $\Gamma$.
} 
and the same for $B, C$, and $D$, with

$$
\begin{array}{ll}
A_{2}=A_{3}=\left[\begin{array}{cccc}
0 & 0 & 0 & 1 \\
0 & 0 & -\nu & 0 \\
0 & \nu & 0 & 0 \\
-1 & 0 & 0 & 0
\end{array}\right], & A_{1}=A_{4}=[0]_{4 \times 4}, \\
B_{2}=B_{3}=\left[\begin{array}{cccc}
0 & 0 & \nu & 0 \\
0 & 0 & 0 & 1 \\
-\nu & 0 & 0 & 0 \\
0 & -1 & 0 & 0
\end{array}\right], & B_{1}=B_{4}=[0]_{4 \times 4}, \\
C_{2}=C_{3}=\left[\begin{array}{cccc}
0 & -\nu & 0 & 0 \\
\nu & 0 & 0 & 0 \\
0 & 0 & 0 & 1 \\
0 & 0 & -1 & 0
\end{array}\right], & C_{1}=C_{4}=[0]_{4 \times 4},
\end{array}
$$

and

$$
D_{4}=\left[\begin{array}{cccc}
-\nu & 0 & 0 & 0 \\
0 & -\nu & 0 & 0 \\
0 & 0 & -\nu & 0 \\
0 & 0 & 0 & 0
\end{array}\right], \quad D_{1}=D_{2}=D_{3}=[0]_{4 \times 4}
$$

The operator $L_{e}$ in (3.1) has the matrix form

$$
L_{e}=\left[l_{i j}(\partial)\right]=A \frac{\partial}{\partial x}+B \frac{\partial}{\partial y}+C \frac{\partial}{\partial z}+D
$$

We also define the space for the enriched variables as

$$
V_{e}=\left\{\underline{v} \in\left[H^{1}(\Omega)\right]^{8}\right\} \text {. }
$$

Following the procedure in [1], we check the polynomials $l_{i j}(\underline{\theta})$. We find that there exist integer weights $s_{i}=0, t_{j}=1, i, j=1,2, \ldots, 8$, such that

$$
\operatorname{deg}\left[l_{i j}(\underline{\boldsymbol{\theta}})\right] \leq s_{i}+t_{j} \text { for } i, j=1,2, \ldots, 8,
$$

where $\underline{\theta}=\left[\begin{array}{lll}x & y & z\end{array}\right]^{t}$ is the spacial variable. We define $l_{i j}^{\prime}$ to be the polynomial with the terms in $l_{i j}$ which are precisely of the order $s_{i}+t_{j}$,

$$
\begin{aligned}
\mathscr{L}(\underline{\boldsymbol{\theta}}) & \equiv \operatorname{det}\left[l_{i j}^{\prime}(\underline{\boldsymbol{\theta}})\right]=\operatorname{det}(x A+y B+z C)=\nu^{4}\left(x^{2}+y^{2}+z^{2}\right)^{4} \\
& \neq 0 \quad \text { for real } \underline{\boldsymbol{\theta}}=\left[\begin{array}{lll}
x & y & z
\end{array}\right]^{t} \neq \underline{0} .
\end{aligned}
$$

By the theory of Agmon, Douglis, and Nirenberg [1], the operator $L_{e}$ defined in (3.1) is an elliptic system and is also uniformly elliptic by the definition given in [20]. In this paper, since we discuss the problem with constant coefficients, the position variable $P$ is dropped. We state the supplementary condition, which is fulfilled for our problem, since we have three independent variables.

Supplementary Condition. $\mathscr{L}(\underline{\theta})$ is of even degree $2 m$. For any pair of linearly independent real vectors $\underline{\boldsymbol{\theta}}$ and $\underline{\boldsymbol{\theta}}^{\prime}$, the polynomial $\mathscr{L}\left(\underline{\boldsymbol{\theta}}+\tau \underline{\boldsymbol{\theta}}^{\prime}\right)$ in the complex variable $\tau$ has exactly $m$ roots with positive imaginary part.

Next, we check the boundary condition to see whether it satisfies the complementing condition.

The operator $R_{e}$ in (3.2) involves a constant matrix of order $4 \times 8$. The order of the boundary operator $R_{e}$ depends on two systems of integer weights, 
in this case the system $t_{j}, j=1,2, \ldots, 8$, already attached to the dependent variables, and a new system $r_{h}, h=1,2,3,4$, of which $r_{h}$ pertains to the $h$ th condition in (3.2). In this paper we simply take $r_{h}=-1, h=1, \ldots, 4$. Let $R_{h j}^{\prime}(\underline{\theta})$ consist of the terms in $R_{h j}(\underline{\theta})$ which are precisely of the order $r_{h}+t_{j}$. There is no difference between $R_{h j}$ and $R_{h j}^{\prime}$ in our problem.

At any point $P$ on a regular portion of $\Gamma$, let $\underline{n}$ denote the outer normal at $P$, and $\underline{\Theta} \neq 0$ any tangent to $\Gamma$. Denote by $\tau_{h}^{+}(\underline{\Theta}), h=1,2,3,4$, the four roots in $\tau$ with positive imaginary part of the characteristic equation $\mathscr{L}(\underline{\boldsymbol{\theta}}+\tau \underline{n})=0$. The existence of these roots is assured by the Supplementary Condition. Set

$$
M^{+}(P, \underline{\boldsymbol{\theta}}, \tau)=\prod_{h=1}^{4}\left(\tau-\tau_{h}^{+}(P, \underline{\boldsymbol{\theta}})\right) .
$$

In our case, $\mathscr{L}(\underline{\boldsymbol{\theta}}+\tau \underline{n})=0$ implies

$$
\left(\left(\theta_{1}+\tau n_{1}\right)^{2}+\left(\theta_{2}+\tau n_{2}\right)^{2}+\left(\theta_{3}+\tau n_{3}\right)^{2}\right)^{4}=0
$$

or $\left(\tau^{2}+1\right)^{4}=0$, so $M^{+}(P, \underline{\Theta}, \tau)=(\tau-i)^{4}$. Let $\left(L^{j k}(\underline{\Theta})\right)$ denote the matrix adjoint to $\left(l_{i j}^{\prime}(\underline{\theta})\right)$,

$$
\left(l_{i j}^{\prime}(\underline{\Theta})\right)=\left[\begin{array}{cccccccc}
0 & 0 & 0 & 0 & 0 & -\nu z & \nu y & x \\
0 & 0 & 0 & 0 & \nu z & 0 & -\nu x & y \\
0 & 0 & 0 & 0 & -\nu y & \nu x & 0 & z \\
0 & 0 & 0 & 0 & -x & -y & -z & 0 \\
0 & -\nu z & \nu y & x & 0 & 0 & 0 & 0 \\
\nu z & 0 & -\nu x & y & 0 & 0 & 0 & 0 \\
-\nu y & \nu x & 0 & z & 0 & 0 & 0 & 0 \\
-x & -y & -z & 0 & 0 & 0 & 0 & 0
\end{array}\right] .
$$

After tedious elementary operations, the adjoint matrix to $\left(l_{i j}^{\prime}(\underline{\theta})\right)$ is seen to be

$$
\left(L^{j k}(\underline{\boldsymbol{\theta}})\right)=\nu^{3}\left(x^{2}+y^{2}+z^{2}\right)^{3}\left[\begin{array}{cccccccc}
0 & 0 & 0 & 0 & 0 & -z & y & \nu x \\
0 & 0 & 0 & 0 & z & 0 & -x & \nu y \\
0 & 0 & 0 & 0 & -y & x & 0 & \nu z \\
0 & 0 & 0 & 0 & -\nu x & -\nu y & -\nu z & 0 \\
0 & -z & y & \nu x & 0 & 0 & 0 & 0 \\
z & 0 & -x & \nu y & 0 & 0 & 0 & 0 \\
-y & x & 0 & \nu z & 0 & 0 & 0 & 0 \\
-\nu x & -\nu y & -\nu z & 0 & 0 & 0 & 0 & 0
\end{array}\right] .
$$

The above-mentioned criterion for the boundary problem (3.1), (3.2) to be coercive is that the following algebraic condition be satisfied.

Complementing Boundary Condition. For any $P \in \Gamma$ and any real, nonzero vector $\underline{\theta}$ tangent to $\Gamma$ at $P$, regard $M^{+}(P, \underline{\theta}, \tau)$ and the elements of the matrix

$$
\sum_{j=1}^{N} R_{h j}^{\prime}(P, \underline{\Theta}+\tau \underline{n}) L^{j k}(P, \underline{\Theta}+\tau \underline{n})
$$


as polynomials in the indeterminate $\tau$. The rows of the latter matrix are required to be linearly independent modulo $M^{+}(P, \underline{\boldsymbol{\theta}}, \tau)$, i.e.,

$$
\sum_{h=1}^{m} C_{h} R_{h j}^{\prime} L^{j k} \equiv 0 \quad\left(\operatorname{Mod} M^{+}\right)
$$

only if the constants $C_{h}$ are all zero.

Without loss of generality, let the tangent vector $\underline{\theta}=\left(\theta_{1}, \theta_{2}, \theta_{3}\right)$ and the normal vector $\underline{n}=\left(n_{1}, n_{2}, n_{3}\right)$ be unit vectors. In our case, $N=8$. From (3.2) and (3.6) we find that the matrix defined in (3.7) for our problem is

$$
\nu^{3}\left(\tau^{2}+1\right)^{3}\left[\begin{array}{cccccccc}
0 & 0 & 0 & 0 & 0 & -m_{3} & m_{2} & \nu m_{1} \\
0 & 0 & 0 & 0 & m_{3} & 0 & -m_{1} & \nu m_{2} \\
0 & 0 & 0 & 0 & -m_{2} & m_{1} & 0 & \nu m_{3} \\
\theta_{3} n_{2}-\theta_{2} n_{3} & \theta_{1} n_{3}-\theta_{3} n_{1} & \theta_{2} n_{1}-\theta_{1} n_{2} & \nu \tau & 0 & 0 & 0 & 0
\end{array}\right] \text {, }
$$

where

$$
\left\{\begin{array}{l}
m_{1}=\theta_{1}+\tau n_{1} \\
m_{2}=\theta_{2}+\tau n_{2} \\
m_{3}=\theta_{3}+\tau n_{3}
\end{array}\right.
$$

Assume the condition (3.8) is fulfilled. In terms of the matrix entries, the condition implies that there are constants $D_{1}, D_{2}, \ldots, D_{8}$ such that

$$
\begin{aligned}
C_{4} \nu^{3}\left(\tau^{2}+1\right)^{3}\left(\theta_{3} n_{2}-\theta_{2} n_{3}\right) & =D_{1} M_{+}(\tau), \\
C_{4} \nu^{3}\left(\tau^{2}+1\right)^{3}\left(\theta_{1} n_{3}-\theta_{3} n_{1}\right) & =D_{2} M_{+}(\tau), \\
C_{4} \nu^{3}\left(\tau^{2}+1\right)^{3}\left(\theta_{2} n_{1}-\theta_{1} n_{2}\right) & =D_{3} M_{+}(\tau), \\
C_{4} \nu^{4}\left(\tau^{2}+1\right)^{3} \tau & =D_{4} M_{+}(\tau), \\
\nu^{3}\left(\tau^{2}+1\right)^{3}\left(C_{2} m_{3}-C_{3} m_{2}\right) & =D_{5} M_{+}(\tau), \\
\nu^{3}\left(\tau^{2}+1\right)^{3}\left(C_{3} m_{1}-C_{1} m_{3}\right) & =D_{6} M_{+}(\tau), \\
\nu^{3}\left(\tau^{2}+1\right)^{3}\left(C_{1} m_{2}-C_{2} m_{1}\right) & =D_{7} M_{+}(\tau), \\
\nu^{4}\left(\tau^{2}+1\right)^{3}\left(C_{1} m_{1}+C_{2} m_{2}+C_{3} m_{3}\right) & =D_{8} M_{+}(\tau) .
\end{aligned}
$$

The roots of the polynomial $M_{+}(\tau)$ have positive imaginary parts with multiplicity 4. On the left-hand sides we have the factor of positive imaginary parts with multiplicity 3 only, hence $D_{1}, D_{2}, \ldots, D_{8}=0$, and $C_{4}=0$. From the last four equations above we have

$$
\begin{gathered}
\left(C_{1}, C_{2}, C_{3}\right)^{T} \times\left(m_{1}, m_{2}, m_{3}\right)^{T}=\underline{0}, \\
\left(C_{1}, C_{2}, C_{3}\right)^{T} \cdot\left(m_{1}, m_{2}, m_{3}\right)^{T}=0 .
\end{gathered}
$$

Since $\left(m_{1}, m_{2}, m_{3}\right)^{T} \not \equiv \underline{0}$, we have $C_{1}=C_{2}=C_{3}=0$, and the Complementing Boundary Condition is indeed satisfied.

By the work in [1], we can now state

Theorem 1. For $l \geq 0$ there is a constant $C>0$ such that

$$
\|\underline{\mathscr{U}}\|_{l+1} \leq C\left(\left\|L_{e} \underline{\mathscr{U}}\right\|_{l}+\left\|R_{e} \underline{\mathscr{U}}\right\|_{l+\frac{1}{2}}+\|\underline{\mathscr{U}}\|_{0}\right) .
$$

It can be shown that the boundary value problem associated with (3.1), (3.2) has a unique solution. Therefore, the term $\|\underline{\mathscr{U}}\|_{0}$ can be dropped from (3.9). If the resulting inequality is applied with $k=0$, we obtain the a priori inequality

$$
\|\underline{\mathscr{U}}\|_{l+1} \leq C\left(\left\|L_{e} \underline{\mathscr{U}}\right\|_{l}+\left\|R_{e} \underline{\mathscr{U}}\right\|_{l+\frac{1}{2}}\right) \text {. }
$$


The inequality (3.10) is crucial for our least squares error analysis. It is interesting to note that (3.10) contains, in particular, the usual shift inequality for the system (1.1). Let $[\underline{u}, p]$ solve $(1.1)$, and let $\underline{\omega}=\operatorname{curl} \underline{u}$. Then $\underline{\mathscr{U}}=[\underline{u}, k=0, \underline{\omega}, p]^{t}$ satisfies $L_{e} \underline{\mathscr{U}}=[\underline{f}, 0, \underline{0}, 0]^{t}$ and $R_{e} \underline{\mathscr{U}}=[\underline{0}, 0]^{t}$. Hence (3.10) yields

$$
\|\underline{u}\|_{l+1}+\|\operatorname{curl} \underline{u}\|_{l+1}+\|p\|_{l+1} \leq C\|\underline{f}\|_{l} .
$$

Since $\operatorname{div} \underline{u}=0,(3.11)$ yields the usual a priori inequality,

$$
\|\underline{u}\|_{l+2}+\|p\|_{l+1} \leq C\|\underline{f}\|_{l},
$$

for solutions of (1.1).

\section{ERROR ESTIMATES}

In this section we will discuss the numerical scheme defined by (2.11). Denote the bilinear form

$$
a(\underline{U}, \underline{V})=\int_{\Omega} L \underline{U} \cdot L \underline{V}+h^{-1} \int_{\Gamma} R \underline{U} \cdot R \underline{V} .
$$

Thus, (2.9) and (2.11) can be reformulated as follows: find $\underline{U} \in V$ (defined by (2.6)) such that

$$
a(\underline{U}, \underline{V})=\int_{\Omega}\left(\frac{f}{0}\right) \cdot L \underline{V} \text { for any } \underline{V} \in V,
$$

and find $\underline{U}^{h} \in V_{r}^{h}$ (defined by (2.7)), such that

$$
a\left(\underline{U}^{h}, \underline{V}^{h}\right)=\int_{\Omega}\left(\frac{f}{0}\right) \cdot L \underline{V}^{h} \quad \text { for any } \underline{V}^{h} \in V_{r}^{h} .
$$

By inspection, $a$ is symmetric and $a(\underline{U}, \underline{U}) \geq 0$. Furthermore, if $a(\underline{U}, \underline{U})=$ 0 , from (3.10) we get $\underline{U}=0$. Hence, the matrix associated with the linear system (2.11) is positive definite.

Combining (4.2), (4.3), we have

$$
a\left(\underline{U}-\underline{U}^{h}, \underline{V}^{h}\right)=0 \text { for any } \underline{V}^{h} \in V_{r}^{h} .
$$

To obtain an error estimate for our least squares method, we shall require an "inverse assumption" on the subspace $V_{r}^{h}$. Inverse assumptions are common in least squares analyses; see, for example, $[2,7]$. The property we need is the existence of a constant $C>0$ such that

$$
\left\|R \underline{V}^{h}\right\|_{1 / 2, \Gamma} \leq C h^{-1 / 2}\left\|R \underline{V}^{h}\right\|_{0, \Gamma} \text { for any } \underline{V}^{h} \in V_{r}^{h} .
$$

Our error estimate is contained in the following theorem.

Theorem 2. Suppose $V_{r}^{h}$ approximates optimally with respect to $r$ and satisfies (4.5). Let $[\underline{u}, p]^{t}$ be the solution of $(1.1)$. Let $\omega=\operatorname{curl} \underline{u}, \underline{U}=[\underline{u}, \underline{\omega}, p]^{t}$, and $\underline{U}^{h} \in V_{r}^{h}$ be the solution of (2.1). Then

$$
\left\|\underline{U}-\underline{U}^{h}\right\|_{1} \leq C h^{r}\|\underline{U}\|_{r+1} .
$$

Proof. Using (3.10) with $l=1,(4.5)$, and (4.1), we have for any $\underline{V}^{h} \in V_{r}^{h}$

$$
\begin{aligned}
\left\|\underline{V}^{h}\right\|_{1}^{2} & \leq C\left(\left\|L \underline{V}^{h}\right\|_{0}^{2}+\left\|R \underline{V}^{h}\right\|_{1 / 2, \Gamma}^{2}\right) \\
& \leq C\left(\left\|L \underline{V}^{h}\right\|_{0}^{2}+h^{-1}\left\|R \underline{V}^{h}\right\|_{0, \Gamma}^{2}\right)=C \cdot a\left(\underline{V}^{h}, \underline{V}^{h}\right) .
\end{aligned}
$$


Applying this inequality to $\underline{U}^{h}-\underline{V}^{h} \in V_{r}^{h}$ and using (4.4), we get

$$
\begin{aligned}
\left\|\underline{U}^{h}-\underline{V}^{h}\right\|_{1}^{2} & \leq C a\left(\underline{U}^{h}-\underline{V}^{h}, \underline{U}^{h}-\underline{V}^{h}\right) \\
& =C\left(a\left(\underline{U}^{h}-\underline{U}, \underline{U^{h}}-\underline{V}^{h}\right)+a\left(\underline{U}-\underline{V}^{h}, \underline{U}^{h}-\underline{V}^{h}\right)\right) \\
& =C a\left(\underline{U}-\underline{V}^{h}, \underline{U}^{h}-\underline{V}^{h}\right) \leq C_{1}\left\|\underline{U}-\underline{V}^{h}\right\|_{1} \cdot\left\|\underline{U}^{h}-\underline{V}^{h}\right\|_{1} .
\end{aligned}
$$

Hence, $\left\|\underline{U}^{h}-\underline{V}^{h}\right\|_{1} \leq C\left\|\underline{U}-\underline{V}^{h}\right\|_{1}$. Using the optimal approximation property of $\underline{V}_{r}^{h}$, we choose $\underline{V}^{h}$ so that $\left\|\underline{U}-\underline{V}^{h}\right\|_{1} \leq C h^{r}\|\underline{U}\|_{r+1}$. Then $\left\|\underline{U}^{h}-\underline{V}^{h}\right\|_{1} \leq$ $C h^{r}\|\underline{U}\|_{r+1}$, and so

$$
\left\|\underline{U}-\underline{U}^{h}\right\|_{1} \leq\left\|\underline{U}-\underline{V}^{h}\right\|_{1}+\left\|\underline{U}^{h}-\underline{V}^{h}\right\|_{1} \leq C h^{r}\|\underline{U}\|_{r+1},
$$

which is the desired result.

\section{BIBLIOGRAPHY}

1. S. Agmon, A. Douglis, and L. Nirenberg, Estimates near the boundary for solutions of elliptic partial differential equations satisfying boundary conditions. II, Comm. Pure Appl. Math. 17 (1964), 35-92.

2. A. K. Aziz, R. B. Kellogg, and A. B. Stephens, Least squares methods for elliptic systems, Math. Comp. 44 (1985), 53-70.

3. A. K. Aziz and J. L. Liu, A weighted least squares method for the backward-forward heat equation, SIAM J. Numer. Anal. 28 (1991), 156-167.

4. I. Babuška, The finite element method with Lagrangian multipliers, Numer. Math. 20 (1973), 179-192.

5. I. Babuška, J. T. Oden, and K. Lee, Mixed-hybrid finite element approximations of second-order boundary value problems, Comput. Methods Appl. Mech. Engrg. 11 (1977), 175206.

6. J. H. Bramble and R. Scott, Simultaneous approximation in scales of Banach spaces, Math. Comp. 32 (1978), 947-954.

7. J. H. Bramble and A. H. Schatz, Least squares for $2 m$ th order elliptic boundary-value problems, Math. Comp. 25 (1971), 1-32.

8. F. Brezzi, On the existence, uniqueness, and approximation of saddle-point problems arising from Lagrange multipliers, RAIRO Anal. Numer. 8 (1974), 129-151.

9. F. Brezzi and J. Douglas, Jr., Stabilized mixed methods for the Stokes problem, Numer. Math. 53 (1988), 225-235.

10. G. F. Carey and B. N. Jiang, Least-squares finite elements for first-order hyperbolic systems, Internat. J. Numer. Mech. Engrg. 26 (1988), 81-93.

11. C.-L. Chang, A finite element method for first order elliptic systems of 3-D, Appl. Math. Comput. 23 (1987), 171-184.

12. C.-L. Chang and M. D. Gunzburger, A subdomain Galerkin/least squares method for firstorder elliptic systems in the plane, SIAM J. Numer. Anal. 27 (1990), 1197-1221.

13. C.-L. Chang and B. N. Jiang, An error analysis of least-squares finite element method of velocity-pressure-vorticity formulation for Stokes problem, Comput. Methods Appl. Mech. Engrg. 84 (1990), 247-255.

14. P. Ciarlet, The finite element method for elliptic problems, North-Holland, Amsterdam, 1978.

15. G. J. Fix, M. D. Gunzburger, and R. A. Nicolaides, On finite element methods of the least squares type, Comput. Math. Appl. 5 (1979), 87-98.

16. G. J. Fix and M. E. Rose, A comparative study of finite element and finite difference methods for Cauchy-Riemann type equations, SIAM J. Numer. Anal. 22 (1985), 250-261.

17. V. Girault and P. A. Raviart, Finite element methods for Navier-Stokes equations, SpringerVerlag, Berlin, 1986. 
18. B. N. Jiang and C.-L. Chang, Least-squares finite elements for Stokes problem, Comput. Methods Appl. Mech. Engrg. 78 (1990), 297-311.

19. B. N. Jiang and L. A. Povinelli, Least-squares finite element method for fluid dynamics, Comput. Methods Appl. Mech. Engrg. 81 (1990), 13-37.

20. C. Miranda, Partial differential equations of elliptic type, 2nd rev. ed. (Zane C. Motteler, translator), Springer-Verlag, Berlin, 1970.

21. P. Neittaamäki and J. Saranen, Finite element approximation of vector fields given by curl and divergence, Math. Methods Appl. Sci. 3 (1981), 328-335.

22. R. Temam, Navier-Stokes equations and nonlinear functional analysis, SIAM, Philadelphia, PA, 1983.

23. W. L. Wendland, Elliptic systems in the plane, Pitman, London, 1979.

24. O. C. Zienkiewicz, The finite element method, Vol. 1, 4th ed., McGraw-Hill, New York, 1989.

25. O. C. Zienkiewicz, D. R. J. Owen, and K. Niles, Least-squares finite element for elasto-static problems-use of reduced integration, Internat. J. Numer. Methods Engrg. 8 (1974), 341358.

Department of Mathematics, Cleveland State University, Cleveland, Ohio 44115

E-mail address: chang@utamat.uta.edu 